W. GÄHLER

GRUNDSTRUKTUREN DER ANALYSIS I 
MATHEMATISCHE LEHRBÜCHER UND MONOGRAPHIEN

HERAUSGEGEBEN VON DER

AKADEMIE DER WISSENSCHAFTEN DER DDR

ZENTRALINSTITUT FUUR MATHEMATIK UND MECHANIK

II. ABTEILUNG

MATHEMATISCHE MONOGRAPHIEN

BAN D 41

GRUNDSTRUKTUREN DER ANALYSIS I

VON

W. GÄHLER

A K A D E M I E-VERLAG.BERLIN

1977 


\section{GRUNDSTRUKTUREN \\ DER ANALYSIS I}

von

\section{WERNER GÄHLER}

Zentralinstitut für Mathematik und Mechanik der Akademie der Wissenschaften der DDR

A K A D E M I E - V E R L A G B E R L I N 
Erschienen im Akademie-Verlag, 108 Berlin, Leipziger Straße 3-4

(c) Akademie-Verlag Berlin 1977

Lizenznummer: 202 . 100/568/77

Gesamtherstellung: VEB Druckerei „Thomas Müntzer", 582 Bad Langensalza

Bestellnummer: 7617905 (6071/1) · LSV 1035

Printed in GDR

DDR 48, - M 